\title{
The Mediterranean diet: science and practice
}

\author{
Walter C Willett* \\ Department of Nutrition, Harvard School of Public Health, Huntington Avenue, Boston, MA 021 15, USA
}

\begin{abstract}
Objective: To provide an overview of research relevant to the Mediterranean diet. Design: Personal perspectives.

Setting: International.

Subject: Populations in Europe, North America, Asia.

Results: Approximately 50 years ago, Keys and colleagues described strikingly low rates of coronary heart disease in the Mediterranean region, where fat intake was relatively high but largely from olive oil. Subsequent controlled feeding studies have shown that compared to carbohydrate, both monounsaturated and polyunsaturated fats reduce LDL and triglycerides and increase HDL cholesterol. Importantly, these beneficial metabolic effects are greater in the presence of underlying insulin resistance. In a detailed analysis within the Nurses' Health Study, trans fat from partially hydrogenated vegetable oils (absent in traditional Mediterranean diets) was most strongly related to risk of heart disease, and both polyunsaturated and monounsaturated fat were inversely associated with risk. Epidemiologic evidence has also supported beneficial effects of higher intakes of fruits and vegetables, whole grains, fish, and daily consumption of moderate amounts of alcohol. Together with regular physical activity and not smoking, our analyses suggest that over $80 \%$ of coronary heart disease, $70 \%$ of stroke, and 90\% of type 2 diabetes can be avoided by healthy food choices that are consistent with the traditional Mediterranean diet.

Conclusion: Both epidemiologic and metabolic studies suggest that individuals can benefit greatly by adopting elements of Mediterranean diets.
\end{abstract}

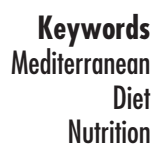

Until very recently in history, Homo sapiens was dependent on foods that were available in its local environment. The fact that populations have survived and developed in vastly different climates and ecological contexts, with correspondingly variable dietary patterns, is a testimony to the remarkable biological and cultural resilience of humans. Although different populations living in an extremely wide range of environments managed to survive through reproductive age, these environments and their corresponding dietary patterns were not necessarily equal in their ability to support long-term health and well-being. The transplantation of plant species across continents and the development of transportation and food preservation technologies have dramatically increased the options of human populations in selecting foods to eat. It is thus natural that the implications of these options for health have become of great interest.

The clinical observations by Dr Grande Covian and others in Southern Europe that rates of coronary heart disease (CHD) were extremely low in this region led to development in the 1960s of the now-famous Seven Countries Study, coordinated by Ancel Keys ${ }^{1}$. Among the 14 populations followed within the seven countries an approximately 10-fold difference in rates was observed, which was well documented by applying standardised diagnostic criteria. This was probably the most fundamentally important contribution of the study as this naturally raised the question as to why these differences existed. For each of the 14 populations that were studied, dietary data were collected in a sample of participants, and it was found that saturated fat was strongly correlated with incidence and mortality rates of CHD. At the same time, Keys and other nutritionists were conducting a series of carefully controlled short-term feeding studies to determine the effects of specific types of fat on serum total cholesterol levels, which had recently been shown to predict CHD incidence in the Framingham cohort and other prospective studies ${ }^{2}$. The results of these controlled feeding studies were summarised in two competing equations by Keys and by Hegsted ${ }^{1,3}$. In both equations, changes in cholesterol were positively related to increases in saturated fat, and inversely related to polyunsaturated fat intake. Together, the Seven Countries Study and the feeding studies had profound effects upon dietary recommendations in the 1960 s and 1970s, whereby the USA and other countries moved to replace saturated fat with polyunsaturated fat. Indeed, during the next 25 years 
polyunsaturated fat in the USA, the UK, Australia, and many other countries approximately doubled, and CHD rates declined by about $50 \%{ }^{2}$. This dietary change was quite fortuitous because the effects of saturated fat and polyunsaturated fat on serum cholesterol are not sufficiently strong to explain either the 10-fold difference in $\mathrm{CHD}$ rates or the $50 \%$ reduction in CHD following recommendations to increase polyunsaturated fat intake. Keys himself noted that the differences in CHD rates were probably due in part to other factors besides saturated fat because the magnitude of these differences, even though the correlation was strong, was too great to be explained by saturated fat intake alone. Considerable discussion has surrounded the contribution of various factors to the decline in CHD, but the large increase in polyunsaturated fat intake is likely to have explained much of this, probably mediated by antithrombotic, antiarrhythmic, and insulin-sensitising effects beyond its effects on serum total cholesterol $^{4}$.

In the late 1980s a major shift in dietary recommendations occurred in Western countries, departing from the message to increase polyunsaturated fat and reduce saturated fat to the idea that all types of fat in the diet should be reduced. The origin of this policy is somewhat hard to determine, but many nutritionists apparently believed that individuals would find it too difficult to understand the differences in types of fat and that it was easier just to tell them to reduce all fat, even though the goal was to reduce saturated fat. This policy appears to have had no empirical basis, as it could well be easier to substitute one type of fat for another rather than to reduce fat in the diet. Also, nutritionists during that period appeared to have forgotten other findings of the Seven Countries Study. Importantly, total fat was not significantly related to $\mathrm{CHD}$ rates because countries with both the lowest and the highest rates of CHD had similar intakes of total fat as a percentage of total energy intake. The obvious difference was, of course, that the nature of the fat was completely different; in the north of Europe this was primarily dairy fat and lard, whereas in Crete (the lowest risk area) and Southern Europe, in general, the fat was largely olive oil.

The belief that fat is inherently unhealthy came to dominate almost all dietary guidelines around the world, including those of the World Health Organization and the United States ${ }^{5-7}$. For example, the US Department of Agriculture's (USDA) 'Food Guide Pyramid' admonished Americans to use fats and oils sparingly, and instead eat large amounts of starch, which in the American diet is largely entirely refined starch and potatoes. Also, the American Heart Association adopted a low-fat campaign and listed as their first dietary advice to use non-fat products $^{8}$. Consequently, grocery store shelves became filled with products such as non-fat yoghurt, non-fat salad dressing, and fat-free cookies and crackers. In most of these products sugar and refined starch replaced fat, and the total amount of calories was similar. Interestingly, the drive to promote fat-free products corresponded with the explosion of obesity in the USA and Northern Europe. The campaign to reduce fat intake may have contributed to the rise in obesity because many nutritionists were telling the public that only calories from fat would make people fat, which could have helped promote the large increase in consumption of carbohydrate that occurred during this period, as people were led to believe that fatfree products were conducive to weight loss. Soberingly, during the late 1980s the rate of decline in CHD mortality slowed and both CHD incidence and mortality due to stroke began to plateau in the USA ${ }^{9,10}$.

Until the 1980s, the investigation of dietary factors that might influence heart disease had almost entirely focused on their impact on total blood cholesterol level. At that time it was realised that high-density lipoprotein (HDL) was an even better predictor of CHD than was total cholesterol, although the direction of this relation was inverse. Similarly, high triglyceride levels were related to heart disease in a positive direction. In a seminal controlled feeding study, Drs Mensink and Katan ${ }^{11}$ compared a diet in which $10 \%$ of energy from saturated fat was replaced either with olive oil or with complex carbohydrates. Thus, the olive oil was nutritionally much like a Mediterranean-type diet, and the complex carbohydrate diet was similar to that being advocated by the American Heart Association and other organisations. In this feeding study total cholesterol levels dropped similarly in both groups, but on the high carbohydrate diet HDL was depressed and triglycerides rose. Thus, all else being equal, one would have predicted a lower rate of CHD with the higher fat/olive oil diet. This finding has been reproduced repeatedly, and has become an important element in understanding the health benefits of the Mediterranean diet.

Subsequently, Mensink and Katan ${ }^{12}$ conducted a controlled feeding study comparing 10\% of energy from saturated fat with $10 \%$ of energy from trans unsaturated fatty acids (trans fat) produced by partial hydrogenation of vegetable oils. Compared with the same percentage of energy from monounsaturated fat, saturated fat increased total cholesterol by $12 \%$, whereas trans fat increased total cholesterol by $6 \%$. This result was used deceptively by parts of the food industry to say that trans fat reduced cholesterol levels compared to saturated fat. While this was true, trans fat reduced total cholesterol in a way that was highly undesirable. Specifically, the increase in low-density lipoprotein (LDL) cholesterol was similar in both diets, but trans fats depressed HDL cholesterol. The change in the ratio of LDL to HDL cholesterol was nearly twice as adverse with the trans fat diet compared to the saturated fat diet. Initially, this finding was controversial, but the finding has been repeatedly reproduced in subsequent studies conducted with various amounts of trans fat in the diet, and there is now consensus that trans fats have adverse effects 
on blood lipids. Overall, the adverse effect of trans fats on the ratio of LDL to HDL cholesterol is approximately twice as bad as the effect of saturated fats on a gram-for-gram basis $^{13}$.

During the last 20 years, research on the aetiology of CHD has shown that the effects of diet may be mediated by many different pathways. Initially, the emphasis was almost entirely on serum total cholesterol but as noted above, we have come to appreciate the importance of HDL, triglycerides, and other lipid fractions. More recently, we now realise that the effects of diet can also be mediated by blood pressure, thrombotic tendency, insulin resistance, antioxidant status, homocysteine, inflammation, endothelial dysfunction, and ventricular arrhythmia. Additional pathways will probably be elucidated in the future. Because of these multiple, potentially synergistic or antagonistic pathways, the effect of diet on heart disease cannot be predicted reliably by examining the effect of diet on one intermediate variable alone. For example, as was shown by the effect of trans fat on serum total cholesterol, we can be completely misled. For this reason it is also desirable to examine the relation between dietary intake and risk of CHD directly. Ideally, this could be done in large randomised trials, but this is practically infeasible in most situations. For this reason our group embarked on a series of large prospective studies to examine the relationship between diet and risk of CHD and other major health outcomes. The first of these studies was the Nurses' Health Study, begun in 1976 under the leadership of Frank Speizer, to examine the relationship between oral contraceptives and breast cancer incidence. This study enrolled 121700 women in 1976, and it quickly became clear that this could provide an opportunity to study the long-term effects of diet, as the participants were highly motivated and could accurately convey information about their health. In this study, we have assessed diet starting in 1980; this information is now updated every four years to take into account changes in the food supply and individual changes in dietary intakes. Thus, we have by now completed seven assessments of diet. During each twoyear follow-up interval, information is also collected about medication use, smoking, physical activity, and other medical events, which provides the ability to control for these factors in the analysis. All cases of heart disease and cancer are documented with medical records. Because the Nurses' Health Study included only women, we enrolled another 52000 men in the Health Professionals Follow-up Study in 1986 using a similar design. The Nurses' Health Study II began in 1989 and included 116000 registered nurses, aged 25-42, to investigate the effects of diet earlier in adult life. Of course, these studies are complex and require the work of many different individuals. Among the current investigators working on these projects are Drs Bernie Rosner, Meir Stampfer, Graham Colditz, David Hunter, JoAnn Manson, Sue Hankinson, Eric Rimm, Edward Giovannucci, Alberto Ascherio, Gary Curhan,
Charlie Fuchs, Fran Grodstein, Michelle Holmes, and Frank Hu. In our first detailed analyses of types of fat and CHD, intakes of each major type of fat were examined in relation to CHD incidence during 14 years of follow-up ${ }^{14}$. Each type of fat was compared with the same percentage of energy from carbohydrate, and each type of fat was adjusted for the influence of the other types of fat. Not surprisingly, trans fat was by far the worst type of fat. Saturated fat was only slightly worse than carbohydrate because exchanging these macronutrients has little effect on the total LDL: HDL ratio. Compared to carbohydrate, monounsaturated fat, and even more so, higher intakes of polyunsaturated fat, were related to lower risk of CHD. The adverse effects of trans fat and the apparent beneficial effects of polyunsaturated fat are substantially greater than might be anticipated by only their effects on HDL and LDL cholesterol. While this at first appeared to be an enigma, more recently we and others have documented that trans fats increase blood levels of lipoprotein(a), the proportion of LDL in the atherogenic form of small particles ${ }^{15}$, triglycerides, and endothelial dysfunction ${ }^{4}$. More recently, an adverse effect of trans fat on inflammatory markers has been documented in both cross-sectional studies and randomised trials ${ }^{16,17}$. This effect on inflammatory markers provides a likely pathway by which the higher risk of type 2 diabetes among women who consume more trans fat may be mediated ${ }^{18}$. The epidemiological data integrate these many effects of trans fats operating through many different pathways, each of them operating in an adverse direction. Similarly, the strong beneficial effects of polyunsaturated fat appear to be mediated by beneficial effects on thrombotic tendency, insulin resistance, and molecular and ventricular arrhythmia ${ }^{4}$. As their influence is in the opposite direction, replacing trans and saturated fat with either mono- or polyunsaturated fat, or a combination of the latter two, will have a large effect on risk of CHD. For example, we found that women in the Nurses' Health Study with high trans fat intake and low polyunsaturated fat intake had about three times the risk of CHD compared to those with low trans fat and high polyunsaturated fat intake ${ }^{14}$.

Much attention has also been given to the specific form of polyunsaturated fat in the diet, the major two forms being the $n-6$ and $n-3$ series. There is now substantial evidence from prospective cohort studies and randomised trials that higher intake of omega-3 fatty acids from fish can reduce risk of sudden death, probably mediated by reduction in occurrence of fatal cardiac arrhythmias ${ }^{4}$. For example, in the Physicians' Health Study we found that eating fish twice a week or more was related to a 50\% lower risk of sudden death compared to those who seldom ate fish ${ }^{19}$. When Dr Albert ${ }^{20}$ and colleagues measured omega- 3 fatty acids in blood, an 80\% lower risk of sudden cardiac death was seen among those with highest levels.

In the Mediterranean region, fish consumption is an integral part of dietary patterns for people living close to 
the sea. However, in inland areas fish consumption has been very low, and $\alpha$-linolenic acid from plants is the major source of omega-3 fatty acids. Less studied than omega-3 fatty acids from fish, lower rates of CHD, both fatal and non-fatal, have been seen with higher intake of $\alpha$-linolenic acid ${ }^{21}$. One of the most compelling studies of diet and CHD was the randomised trial conducted in Lyon, France, by de Lorgeril et $a l^{22}$. In that study the experimental group was assigned to a diet designed to mimic nutritionally the composition of the traditional Cretan diet. This diet was high in $\alpha$-linolenic acid (from rapeseed oil, primarily) and olive oil; the amounts of red meat and dairy products were low. The control diet was similar to the low-fat diet suggested by the American Heart Association. Over the course of five years, a $70 \%$ reduction in the risk of death or recurrent cardiovascular disease was seen in the Mediterranean-type diet compared to the control group. Notably, this benefit emerged just a few months after starting, presumably due to the anti thrombotic and antiarrhythmic pathways, because there was little difference between diets in lipid levels.

Until recently, dietary carbohydrates had been minimally studied in comparison to the huge literature on fat and type of fat, although studies had examined fibre in relation to risk of CHD. In prospective studies, intakes of fibre from whole-grain products have been consistently related to lower risk of $\mathrm{CHD}^{4}$. For example, in the Health Professionals' Follow-up Study we observed a 30\% lower risk among men with the highest level of intake. The beneficial effect may be mediated by not just fibre per se, but the many minerals and vitamins contained in whole grains that are stripped away when these grains are refined. In addition to their effects on risk of CHD, whole-grain, high-fibre forms of cereal products have been consistently related to lower risk of type 2 diabetes ${ }^{23}$. The apparent benefit of whole-grain cereal products appears to be, in part, due to the higher intake of cereal fibre, and also to greater ingestion of carbohydrates in a low glycaemic form.

One form of carbohydrate intake that is devoid of fibre and has a high glycaemic load is sugar-sweetened soda beverages, which are now consumed in large amounts almost worldwide, although these were absent in traditional Mediterranean diets. We have recently reported that high soda consumption is related to both weight gain and, independently, risk of type 2 diabetes ${ }^{24}$. One important finding during the past decade was that the adverse effects of high glycaemic load (reflecting both glycaemic index and the amount of carbohydrate) is influenced by the underlying degree of insulin resistance $^{25}$. In large epidemiologic studies, direct measurements of insulin resistance are not practical, but body mass index (BMI) serves as an indirect but useful surrogate variable. In many instances we have confirmed that the adverse effects of high glycaemic load are modest or nonexistent in lean, active individuals, but among those with average or above-average BMI, high amounts of high-glycaemic carbohydrates have been related to $\mathrm{CHD}^{26}$ and stroke ${ }^{27}$. This finding has important implications because many societies are moving from active peasant lifestyles to a more sedentary way of existence in which body weight tends to increase. Thus, the same diet that might have been tolerated well in a more traditional lifestyle may have adverse effects in the new context.

The most persistent argument used to advocate diets high in starch and low in fat has been that this would fight obesity $^{28}$. However, the evidence to support this has usually been based on studies that were of only a few months' duration. In randomised trials of one year or longer, no overall benefit of reducing the percentage of energy from fat has been seen ${ }^{29}$.

During the last decade we have learned much about the various aspects of diet that can reduce the risk of CHD. Although most of these studies have not been conducted among populations consuming Mediterranean diets, the findings consistently support the healthfulness of the traditional Mediterranean dietary pattern. To examine the potential quantitative impact of healthy dietary choices and lifestyle factors on risk of CHD, Stampfer et al. ${ }^{30}$ used data from 14 years of follow-up in the Nurses' Health Study. In this analysis a low-risk group was identified as consisting of non-smokers, BMI $<25 \mathrm{~kg} / \mathrm{m}^{2}, 1 / 2$ hour/day or more of moderate-to-vigorous activity such as brisk walking, being in the upper two quartiles of a dietary score based on low trans fat, high polyunsaturated-to-saturated fat ratio, low glycaemic load, high cereal fibre intake, fish consumption twice/week or more, high folate intake, and moderate alcohol (1/2 drink per day or more). Surprisingly, only $3.1 \%$ of the women in the population fell into this category of low risk. However, we calculated that if everyone had assumed this low-risk behaviour, over $80 \%$ of premature CHD events would have been avoided ${ }^{30}$. Although this number of over $82 \%$ seemed high to many, this should be no surprise given that low rates of CHD in traditional Crete were approximately 80-90\% lower than those in the United States at that time. Our analysis has confirmed that a large majority of CHD incidence is preventable, and also has identified specific aspects of diet and lifestyle that can achieve this reduction in risk. Importantly, these do not demand adopting a lifestyle of deprivation or intense physical labour, as they require only moderate changes that can be potentially achievable by almost everyone. In a similar analysis, we examined the percentage of type 2 diabetes incidence that is potentially preventable ${ }^{18}$. Interestingly, almost all of these same behaviours were also related to risk of type 2 diabetes, except that folic acid and fish did not enter the definition of 'low risk' as they were not related to risk of type 2 diabetes. In this analysis we observed that over $92 \%$ of type 2 diabetes is potentially avoidable. Trichopoulou et $a l^{31}$ have also found that a dietary score reflecting the traditional Mediterranean dietary pattern was related to lower risk of total mortality in Greek men and women. 
The series of studies described above has added to concerns that the dietary messages embedded in most recommendations, such as those of the USDA Food Guide Pyramid, do not provide consumers with adequate guidance. For example, they ignore critical differences in the type of fat, overemphasise starch intake without paying adequate attention to the degree of refining, and advocate diets that are high in red meat and dairy. In analyses conducted by McCullough et al. ${ }^{32,33}$, we used the Healthy Eating Index, developed by the USDA to quantify adherence to the Food Guide Pyramid, and the US Dietary Guidelines. After accounting for smoking and other lifestyle factors, there was essentially no relationship between adherence to these dietary guidelines and risk of major chronic disease in women, and only a slightly lower risk in men. However, a Revised Healthy Eating Index that took into account the type of fibre and the form of fat did better predict occurrence of major chronic disease in both men and women ${ }^{34}$. Notably, though, the benefit appeared to be primarily for cardiovascular disease, whereas there was actually little relationship between this revised index and risk of cancer. Nevertheless, in these and other cohorts, lean body weight and regular physical activity was related to lower risk of cancer as well as cardiovascular disease ${ }^{35}$.

The results of these analyses have emphasised that the focus on reduction in total fat intake has distracted attention from aspects of diet that could have important public health benefits. Fortunately, during the last few years a shift away from an emphasis on low-fat diets has been occurring. For example, the American Heart Association has recently noted that 'The diet high in unsaturated fat (up to $35 \%$ calories from unsaturated fat plus up to $10 \%$ of calories of saturated fat) can be a viable alternative to a diet that is low in total fat ${ }^{8}$. A 2002 Institute of Medicine/National Academy of Sciences report emphasised the type of fat rather than the amount of fat, and allowed intakes of up to $35 \%$ of calories ${ }^{36}$. Similarly, the 2005 US Dietary Guidelines have moved away from an emphasis on low fat intake, suggesting a range of $20-35 \%$ of energy from fat and strongly emphasising the need to reduce trans fat intake ${ }^{37}$.

In many ways, the recent Dietary Guidelines have only now been catching up with the actual behaviour of individuals in the United States. For example, olive oil consumption has increased many-fold from the time when it was only a minor occupant of grocery store shelves. Also, the countries of Northern Europe that had the highest rates of cardiovascular disease in the world during Keys' original studies have adopted many of the features of the Mediterranean diet, in particular a dramatic reduction in saturated fat and increase in unsaturated fat. As expected, rates of $\mathrm{CHD}$ have plummeted and life expectancy of adults has increased dramatically. In contrast, lifestyles have changed in the Mediterranean area toward those of Northern Europe. The changes have not been in diet alone as the role of physical activity has also clearly declined. Although this change has not been well documented statistically, it has been clear to those who have watched the evolution of the Greek countryside over time. In the 1960s and 1970s during my visits there, much of the work was done by hand, including the harvesting and threshing of grain. Travel was often by foot or donkey. During my later visits back to the same areas, the way of travel changed from traditional forms to 3-wheeled motor vehicles, motorcycles, and cars. The stone threshing circles in the countryside that were actively used in the 1960s have been overgrown and are now becoming 'archaeological artefacts'. Formal surveys are not even needed to see that overweight and obesity have greatly increased. Although Greece had the best life expectancy in the world in the $1960 \mathrm{~s}^{38}$, life expectancy from 60 years is now highest in the Scandinavian countries, Japan, and Switzerland ${ }^{39}$. Ironically, the major benefactors in the research on the Mediterranean lifestyle and health have not been the Mediterranean countries. Fortunately, the need to preserve and promote the traditional Mediterranean diet has been recognised in the Mediterranean area itself, and this is manifested in part by the symposium. Many years of scientific investigation can allow us to conclude that these efforts, if successfully implemented, will profoundly benefit the health of everyone.

\section{References}

1 Keys A. Seven Countries: A Multivariate Analysis of Death and Coronary Heart Disease. Cambridge, MA: Harvard University Press, 1980.

2 Willett WC. Nutritional Epidemiology, 2nd ed. New York: Oxford University Press, 1998.

3 Hegsted DM. Serum-cholesterol response to dietary cholesterol: a re-evaluation. American Journal of Clinical Nutrition 1986; 44: 299-305.

4 Hu FB, Willett WC. Optimal diets for prevention of coronary heart disease. Journal of the American Medical Association 2002; 288(20): 2569-78.

5 US Department of Agriculture, US Department of Health and Human Services. Nutrition and Your Health: Dietary Guidelines for Americans, 5th ed. Home and Garden Bulletin No. 232, Washington, DC: US Government Printing Office, 2000.

6 World Health Organization. The World Health Report 2002: Reducing Risks, Promoting Healthy Life. Geneva: WHO, 2002.

7 World Health Organization. Diet, Nutrition and the Prevention of Chronic Disease. WHO Technical Report series 916. Geneva: WHO, 2003.

8 American Heart Association. Dietary guidelines for healthy American adults. Circulation 1996; 94: 1795-800.

9 Cooper R, Cutler J, Desvigne-Nickens P, Fortmann SP, Friedman L, Havlik R, et al. Trends and disparities in coronary heart disease, stroke, and other cardiovascular diseases in the United States: findings of the National Conference on Cardiovascular Disease Prevention. Circulation 2000; 102: 3137-47.

10 Rosamond WD, Chambless LE, Folsom AR, Cooper LS, Conwill DE, Clegg L, et al. Trends in the incidence of 
myocardial infarction and in mortality due to coronary heart disease, 1987 to 1994. New England Journal of Medicine 1998; 339: 861-7.

11 Mensink RP, Katan MB. Effect of monounsaturated fatty acids versus complex carbohydrates on high-density lipoprotein in healthy men and women. Lancet 1987; 1: 122-5.

12 Mensink RPM, Katan MB. Effect of dietary trans fatty acids on high-density and low-density lipoprotein cholesterol levels in healthy subjects. New England Journal of Medicine 1990; 323: 439-45.

13 Ascherio A, Katan MB, Zock PL, Stampfer MJ, Willett WC. Trans fatty acids and coronary heart disease. New England Journal of Medicine 1999; 340: 1994-8.

14 Hu FB, Stampfer MJ, Manson JE, Rimm E, Colditz GA, Rosner $\mathrm{BA}$, et al. Dietary fat intake and the risk of coronary heart disease in women. New England Journal of Medicine 1997; 337: 1491-9.

15 Mauger JF, Lichtenstein AH, Ausman LM, Jalbert SM, Jauhiainen M, Ehnholm C, et al. Effect of different forms of dietary hydrogenated fats on LDL particle size. American Journal of Clinical Nutrition 2003; 78(3): 370-5.

16 Mozaffarian D, Pischon T, Hankinson SE, Rifai N, Joshipura $\mathrm{K}$, Willett WC, et al. Dietary intake of trans fatty acids and systemic inflammation in women. American Journal of Clinical Nutrition 2004; 79(4): 606-12.

17 Baer DJ, Judd JT, Clevidence BA, Tracy RP. Dietary fatty acids affect plasma markers of inflammation in healthy men fed controlled diets: a randomized crossover study. American Journal of Clinical Nutrition 2004; 79(6): 969-73.

$18 \mathrm{Hu} \mathrm{FB}$, Manson JE, Stampfer MJ, Colditz G, Liu S, Solomon CG, et al. Diet, lifestyle, and the risk of type 2 diabetes mellitus in women. New England Journal of Medicine 2001; 345: 790-7.

19 Albert CM, Hennekens CH, O'Donnell CJ, Ajani UA, Carey VJ, Willett WC, et al. Fish consumption and risk of sudden cardiac death. Journal of the American Medical Association 1998; 279: 23-8.

20 Albert CM, Campos H, Stampfer MJ, Ridker PM, Manson JE, Willett WC, et al. Blood levels of long-chain n-3 fatty acids and the risk of sudden death. New England Journal of Medicine 2002; 346(15): 1113-8.

21 Baylin A, Kabagambe EK, Ascherio A, Spiegelman D, Campos H. Adipose tissue alpha-linolenic acid and nonfatal acute myocardial infarction in Costa Rica. Circulation 2003; 107(12): 1586-91.

22 de Lorgeril M, Salen P, Martin J-L, Monjaud I, Delaye J, Mamelle N. Mediterranean diet, traditional risk factors, and the rate of cardiovascular complications after myocardial infarction: final report of the Lyon Diet Heart Study. Circulation 1999; 99: 779-85.

23 Hu FB, van Dam RM, Liu S. Diet and risk of type II diabetes: the role of types of fat and carbohydrate. Diabetologia 2001, 44(7): $805-17$

24 Schulze MB, Manson JE, Ludwig DS, Colditz GA, Stampfer MJ, Willett WC, et al. Sugar-sweetened beverages, weight gain, and incidence of type 2 diabetes in young and middle-aged women. Journal of the American Medical Association 2004; 292(8): 927-34

25 Jeppesen J, Schaaf P, Jones G, Zhou MY, Chen YD, Reaven GM. Effects of low-fat, high-carbohydrate diets on risk factors for ischemic heart disease in postmenopausal women. American Journal of Clinical Nutrition 1997; 65 $1027-33$.

26 Liu S, Willett WC, Stampfer MJ, Hu FB, Franz M, Sampson L, et al. A prospective study of dietary glycaemic load, carbohydrate intake, and risk of coronary heart disease in US women. American Journal of Clinical Nutrition 2000; 71: $1455-61$

27 Oh K, Hu FB, Cho E, Rexrode KM, Stampfer MJ, Manson JE, et al. Carbohydrate intake, glycaemic index, glycaemic load, and dietary fiber in relation to risk of stroke in women. American Journal of Epidemiology 2005; 161(2): 161-9.

28 Bray GA, Popkin BM. Dietary fat intake does affect obesity! American Journal of Clinical Nutrition 1998; 68: 1157-73.

29 Willett WC, Leibel RL. Dietary fat is not a major determinant of body fat. American Journal of Medicine 2002; 113(Suppl 9B): $47 \mathrm{~S}-59 \mathrm{~S}$

30 Stampfer MJ, Hu FB, Manson JE, Rimm EB, Willett WC Primary prevention of coronary heart disease in women through diet and lifestyle. New England Journal of Medicine 2000; 343: 16-22.

31 Trichopoulou A, Costacou T, Bamia C, Trichopoulos D. Adherence to a Mediterranean diet and survival in a Greek population. New England Journal of Medicine 2003; 348 2599-608.

32 McCullough ML, Feskanich D, Rimm EB, Giovannucci EL, Ascherio A, Variyam JN, et al. Adherence to the Dietary Guidelines for Americans and risk of major chronic disease in men. American Journal of Clinical Nutrition 2000; 72 $1223-31$

33 McCullough ML, Feskanich D, Stampfer MJ, Rosner BA, Hu FB, Hunter DJ, et al. Adherence to the Dietary Guidelines for Americans and risk of major chronic disease in women. American Journal of Clinical Nutrition 2000; 72(5): 1214-22.

34 McCullough ML, Feskanich D, Stampfer MJ, Giovannucci EL, Rimm EB, Hu FB, et al. Diet quality and major chronic disease risk in men and women: moving toward improved dietary guidance. American Journal of Clinical Nutrition 2002; 76(6): 1261-71.

35 International Agency for Research on Cancer. Weight Control and Physical Activity. Vol. 6. Lyon: IARC Press, 2002.

36 Institute of Medicine. Dietary Reference Intakes for Energy, Carbohydrate, Fiber, Fat, Fatty Acids, Cholesterol, Protein, and Amino Acids (Macronutrients). A Report of the Panel on Macronutrients, Subcommittees on Upper Reference Levels of Nutrients and Interpretation and Uses of Dietary Reference Intakes, and the Standing Committee on the Scientific Evaluation of Dietary Reference Intakes. Washington, DC: National Academy of Sciences, 2002. Available at http://www.nap.edu/catalog/10490.html.

37 US Department of Health and Human Services, US Department of Agriculture. Dietary Guidelines for Americans, 6th ed. Washington, DC. US Government Printing Office, January 2005.

38 Willett WC. Diet and health: What should we eat? Science 1994; 264: 532-7.

39 World Health Organization. The World Health Report 2003 : Shaping the Future. Geneva: WHO, 2003. 